\title{
Plant-Derived Isoquinoline Alkaloids that Target Ergosterol Biosynthesis Discovered by Using a Novel Antifungal Screening Tool
}

\author{
Siu Wah Wong-Deyrup ${ }^{1, \dagger}$, Xun Song ${ }^{1,2 \dagger}$, Tsz-Wai Ng', Xiu-Bin Liu ${ }^{3,4}$, Jian-Guo Zeng, ${ }^{3,4}$, Zhi-Xing \\ Qing, ${ }^{3,}$, Stephen T. Deyrup ${ }^{5}$, Zhen-Dan He2, , ,Hong-Jie Zhang1, \\ 1 School of Chinese Medicine, Hong Kong Baptist University, Hong Kong, P. R. China; \\ moonie_815@yahoo.com (S.W.); 13480143@life.hkbu.edu.hk (X.S.); wai64@yahoo.com.hk (T.N.) \\ 2 School of Pharmaceutical Science, Health Science Center, Shenzhen University, Shenzhen, P. R. China; \\ hezhendan@szu.edu.cn (Z.H.) \\ 3 Hunan Provincial Key Laboratory of Crop Germplasm Innovation and Utilization and National Chinese \\ Medicinal Herbs Hunan Technology Center, Hunan Agricultural University, Changsha 410128, P. R. \\ China; 87135168@qq.com (X.L.); zengjianguo@hunau.net (J.Z.); 719764893@qq.com (Z.Q.) \\ 4 Hunan Co-Innovation Center for Utilization of Botanicals Functional Ingredients, Hunan University of \\ Chinese Medicine, Changsha 410208, P. R. China \\ 5 Department of Chemistry and Biochemistry, Siena College, Loudonville NY 12211 USA; \\ sdeyrup@siena.edu (S.D.) \\ * Correspondence: zhanghj@hkbu.edu.hk (H.Z.), Tel. +852-3411-2956; hezhendan@szu.edu.cn (Z.H.) \\ + These authors contributed equally.
}

\begin{abstract}
The ergosterol pathway is a prime antifungal target. The minimum inhibitory concentration (MIC) assay is a simple research tool that determines the lowest concentration at which a novel antimicrobial with limited scope to determine the mechanism of action for a drug. In this study, we show that by adding hydrogen peroxide, an oxidative stressor, or glutathione (GSH), an antioxidant, to modify a commonly performed MIC assay allowed us to screen selectively for new antifungal drugs that target ergosterol biosynthesis in fungi. A human pathogen, Microsporum gypseum, was used as a test organism. When exposed to ergosterol targeting drugs, the hydrogen peroxide treatment significantly decreased fungal survival by reducing ergosterol in the cell wall, whereas GSH increased survival of M. gypseum. Further, by performing a series of experiments with M. gypseum, it was determined that the oxidative stress from hydrogen peroxide causes cell death at different developmental stages. These findings allow us to describe a simple, high-throughput method for simultaneously screening new antifungal drugs for activity and effects on the ergosterol pathway. By using this tool, two isoquinoline alkaloids were discovered to be potent inhibitors of ergosterol biosynthesis by reducing the amount of ergosterol without affecting the expression of $1,3-\beta$-glucan.
\end{abstract}

Keywords: Fungi, ergosterol pathway, dermatophytes, chelerythrine, sanguinarine

\section{Introduction}

Dermatophytes are among the most common pathogenic fungi that infect keratinized tissues such as skin, hair, and nails. About $25 \%$ of the world's population have been infected at least once in its lifetime according to the World Health Organization (WHO) [1]. Although the infection is not lifethreatening for healthy individuals, its infection rate increases with age and causes severe symptoms in immunocompromised individuals [2-4]. Meanwhile, the best antifungal allylamine drugs only achieve a $70 \%$ cure rate due to drug resistance that develops over time [5]. The discovery of drugs for treatment of fungal infections is particularly difficult as pathogenic fungi are eukaryotic like mammalian cells. Some patients, such as pregnant women, are also unsuitable to be treated. Even though azoles have shown to be quite effective in treating infections, they are unfortunately 
hepatotoxic [6]. Amphotericin B which disrupts osmotic integrity of the cell membrane through sterol binding also causes significant nephrotoxicity [7]. Because of the narrow therapeutic window, drug resistance, toxicity, and detrimental drug-drug interactions, there is an urgent need to discover novel antifungal agents for future drug development.

Recent antifungal drugs that are available to combat infections are in the classes of azole, allylamine, morpholine, hydroxypyrimidine, and polyene. Each class has a unique mechanism of action, azoles inhibit the synthesis of ergosterol; polyenes bind to ergosterol; allylamine accumulates squalene (upstream of the ergosterol biosynthesis pathway); hydroxypyrimidine impairs DNA replication; and morpholine depletes ergosterol by inhibiting d14-sterol reductase activity [6]. The ergosterol biosynthetic pathway is critical to fungal survival as it is optimized to protect the fungi against mechanical and oxidative stress [8]. The fungal cell wall is the primary defense to external oxidative stressors, such as hydrogen peroxide and paraquat [9]. Ergosterol has been a target of interest on oxidative stress studies because it is the most abundant sterol in fungal cell membranes [10]. Thus, the ergosterol biosynthetic pathway has a strong implication for fungus survival and drug targeting.

Minimal inhibitory concentration (MIC) is a straightforward process to test the susceptibility of the drug activity candidates by measuring cell density on a 96-well microplate. Sorbitol has been added in an MIC assay to screen for antifungal inhibitors but does not distinguish the mode of action [11]. Escalante et al. used the addition of ergosterol in the medium to screen drugs that bind to ergosterol and inhibit the cell membrane formation [12]. Both methods were combined to screen for chemicals that affect ergosterol and cell wall in timely fashion [11]. By introducing an oxidative stress, such as hydrogen peroxide $\left(\mathrm{H}_{2} \mathrm{O}_{2}\right)$, and an antioxidant [13], such as reduced glutathione (GSH), into MIC assays, we developed a simple drug screening method specifically targeting the ergosterol biosynthetic pathway. This is the first example using MIC to determine the mode of action by screening antifungal drugs in different genera of fungi.

Three genera of fungi, Trichophyton, Microsporum, and Epidermophyton, account for most dermatophyte infections. In hot and humid climates like India, $98.65 \%$ cases of dermatophyte infection is found to be Trichophyton spp. followed by Microsporum gypseum (1.35\% cases). There was no case found to have Epidermophyton spp. [14]. In southern China, Trichophyton spp accounted for $63.8 \%$ of identifiable agents caused Tinea pedis [15]. In another study, T. rubrum (zoophilic) accounting for at least $60 \%$ of all superficial fungal infections in human while M. gypseum (geophilic) is found in about $17 \%$ of the patients [16]. Both classes of fungi account for a large proportion of cases of infection. Due to the infection prevalence of T. rubrum and M. gypseum, they are considered as good models for developing a screening tool for both zoophilic and geophilic pathogenic fungi.

Based on this novel screening tool, two antifungal isoquinoline alkaloids isolated from a medicinal plant (Macleaya cordata) were discovered from our natural products bank. We demonstrated their antifungal activity in vitro, and further validated the therapeutic effects of topical application in guinea pig dermatophytosis models.

\section{Materials and methods}

\subsection{Minimal inhibitory concentration (MIC) assay}

Trichophyton rubrum (MYA4438) and Microsporum gypseum (14683) were purchased from ATCC. MICs of various drugs: amphotericin B (AB, Sigma, St Louis, MO, USA), amorolfine (AF, Sigma, St Louis, MO, USA), bifonazole (BFZ, Sigma, St Louis, MO, USA), butenafine (BF, St Louis, MO, USA), ciclopirox (CF, Sigma, St Louis, MO, USA), griseofulvin (GF, St Louis, MO, USA), itraconazole (ITZ, Sigma, St Louis, MO, USA), miconazole (MCZ, Sigma, St Louis, MO, USA), terbinafine (TF, Sigma, St Louis, MO, USA), and tioconazole (TAZ, Sigma, St Louis, MO, USA) were obtained. The assay follows a broth micro-dilution method from the Clinical and Laboratory Standards Institute approved standard M38-A [17]. All the drugs were diluted in dimethyl sulfoxide (DMSO, Sigma, St Louis, MO, USA) and stored at $4^{\circ} \mathrm{C}$.

Stock inoculum was prepared from a 7-day culture grown on potato dextrose agar (PDA, BD, Radnor, PA, USA) at $28^{\circ} \mathrm{C}$ to induce sporulation. The colonies were removed from the agar with 10 $\mathrm{mL}$ sterile distilled water. The conidia and hyphal fragments were then filtered from a Whatman 
paper resulting in an inoculum composed mainly of spores. The spores in the inoculum were stained by lactophenol blue solution (Sigma, St Louis, MO, USA), and counted with a hemocytometer using light microscopy (100X). All inoculum were adjusted to a final concentration at $5 \times 10^{4} \mathrm{spore} / \mathrm{mL}$ with $75 \%$ potato dextrose broth (PDB, BD, Radnor, PA, USA) and 0.5\% DMSO. Polystyrene 96-well flatbottomed wells microtiter plates were used for observing the fungal growth by measuring the cell density at $600 \mathrm{~nm}\left(\mathrm{OD}_{600}\right)$. Each sample was inoculated with $190 \mu \mathrm{L}$ of the diluted inoculum suspensions with a multichannel pipette into a 96-well microplate. Aliquots of $10 \mu \mathrm{L}$ of the 2-fold drug dilutions of antifungal chemicals were added into the media. The microplates were incubated at $28^{\circ} \mathrm{C}$ and the $\mathrm{OD}_{600}$ was measured with Perkin Elmer Ensight at three days after the treatment for M. gypseum and seven days after the treatment for T. rubrum. MIC values for each antifungal drug were determined by the lowest drug concentration with no detectable fungal growth after three or seven days of treatment. The MIC assays of biological samples were performed in triplicate.

Four different inoculum suspension conditions were applied which includes control, sorbitol, GSH (Sigma, St Louis, MO, USA) and hydrogen peroxide $\left(\mathrm{H}_{2} \mathrm{O}_{2}\right)$ (Sigma, St Louis, MO, USA). Their roles were to induce oxidative stress/protection in fungus. The final concentration of $0.8 \mathrm{M}$ sorbitol was chosen according to literature values [11], whereas the concentrations of GSH (5 g/L) and $\mathrm{H}_{2} \mathrm{O}_{2}$ $(200 \mu \mathrm{M})$ were determined by concentration optimization. Sorbitol has been shown to act as an osmoprotectant that is commonly used in MIC assay to give similar or higher MIC values [11]. GSH protects cells from oxidative damages [18]; and is a standard component for MIC screening. $\mathrm{H}_{2} \mathrm{O}_{2}$, however, is reactive oxygen species (ROS) that induces oxidative stress in cells [19], which has not been reported to be used for MIC screening.

\subsection{Confocal imaging}

T. rubrum and M. gypseum stock inoculum were prepared from a 7-day culture grown on PDA at $28^{\circ} \mathrm{C}$ to induce sporulation. The method to remove and the spores count were described in this paper earlier. Aliquots of $10 \mu \mathrm{L}$ of the $1 / 2$ MIC of antifungal drug concentration were added into each suspension. One set of culture was prepared in the presence of $200 \mu \mathrm{M} \mathrm{H}_{2} \mathrm{O}_{2}$ while the other had none. The suspensions were incubated at $28^{\circ} \mathrm{C}$ for three days. The culture was then centrifuged at 4,000 rpm for 10 minutes. The supernatant was removed and the fungi were rinsed with GlucoseHEPES (GH) solution as instructed on the LIVE/DEAD yeast viability kit (Invitrogen, Waltham, MA, USA). The concentration of Fun- 1 dye and Calcofluor white were $3 \mu \mathrm{M}$ and $25 \mu \mathrm{M}$, respectively. Confocal images of fungi were compared between the presence and absence of $\mathrm{H}_{2} \mathrm{O}_{2}$ under different drug conditions. Image J software was used to measure the fluorescence intensity and distribution [20]. The average of intensity and standard deviation were calculated and graphed.

\subsection{Amplex red hydrogen peroxide assay}

T. rubrum and M. gypseum stock inoculum were prepared from a 7-day culture grown on PDA at $28^{\circ} \mathrm{C}$ to induce sporulation. Spore suspensions were made as described above. Aliquots of $10 \mu \mathrm{L}$ of the antifungal drug at a concentration of half the MIC value were added into each suspension. A 96-well microplate for fluorescence-based assay (Invitrogen, Waltham, MA, USA) was used for both measurement of fungal growth at $\mathrm{OD}_{600}$ and of fluorescence assay with Amplex Red. The suspensions were incubated at $28^{\circ} \mathrm{C}$ for three days. The instructions of Amplex Red Hydrogen Peroxide Assay (Invitrogen, Waltham, MA, USA) were followed. The culture was centrifuged at 4,000 rpm for 10 minutes. The supernatant was removed and then the fungi were rinsed with $1 \mathrm{X}$ reaction buffer twice. One set of fungi was incubated in $10 \mathrm{mM} \mathrm{H}_{2} \mathrm{O}_{2}$ while the other had none for 2 hours at room temperature. Amplex Red was added and incubated in the dark for 30 minutes prior to measurement. Meanwhile, a standard curve for $\mathrm{H}_{2} \mathrm{O}_{2}$ and Amplex Red reaction buffer were prepared.

Since the classes of drugs that inhibit biosynthesis of ergosterol and accumulates lanosterol and squalene were shown to be sensitive to hydrogen peroxide, an Amplex Red hydrogen peroxide assay was used to determine how ergosterol, lanosterol, or squalene interact with hydrogen peroxide differently. Ergosterol (Sigma, St Louis, MO, USA) and lanosterol (Sigma, St Louis, MO, USA) were initially dissolved in $100 \%$ ethanol while squalene (Sigma, St Louis, MO, USA) was dissolved in acetone. Those solvents alone were also measured as backgrounds for the assay. Amplex Red reaction buffer was added into each well containing fungi, the standards, and the controls after the 2-hour of 
incubation. The mixture was incubated further at room temperature in the dark for 30 minutes. The level of fluorescence was then measured using Ensight (Perkin Elmer) shaking the plate at $60 \mathrm{rpm}$ for 10 seconds prior to the measurement at the excitation of $530 \mathrm{~nm}$ and the emission at $590 \mathrm{~nm}$. The solvents that were used for dissolving sterols have been shown to not affect the Amplex Red measurement (data not shown). These assays of biological samples were performed in triplicate.

\subsection{Normalization of hydrogen peroxide concentration with cell density}

Due to the nature of tangled mycelia in our fungi, it is difficult to dilute and obtain a uniform fungal culture across the assay. Thus, it is necessary to normalize the concentration of hydrogen peroxide based on fungal cell density $\left(\mathrm{OD}_{600}\right)$. In order to do this, the finalized $\mathrm{OD}_{600}$ was adjusted with a background subtraction of potato dextrose broth (PDB) in the wells. The standard curve for hydrogen peroxide should produce $\mathrm{R}^{2}>0.95$. The equation to calculate the concentration of hydrogen peroxide was obtained from the linear equation of the standard curve. The normalized hydrogen peroxide concentration was determined by the concentration of hydrogen peroxide divided by the finalized OD 600 which represents the amount of hydrogen peroxide per one unit of cell density.

\subsection{Mycelia growth under oxidative stress}

The inoculum was prepared as described previously in this paper. The suspensions were incubated at $28^{\circ} \mathrm{C}$ and their growth rate was monitored under a $120 \mathrm{X}$ amplification in stereomicroscope (Leica) every 24 hours. The images were taken after 48 hours of incubation from a 96-well microplate to observe the different modes of germination. The growth of hyphae in hydrogen peroxide was measured by comparing that with the control in an area of $20 \mathrm{~mm} \times 20 \mathrm{~mm}$.

\subsection{MIC screen for detecting ergosterol pathway antifungal activity}

The preparation of the MIC assay was performed as described above. The compounds from our natural products bank were diluted in DMSO. M. gypseum with $5 \times 10^{4}$ spores $/ \mathrm{mL}$ was added to a series of 2-fold dilutions of the chemicals with final concentrations ranging from 0 to $100 \mathrm{mM}$ in $0.5 \%$ DMSO in PDB. After incubating the fungal mixture at $28^{\circ} \mathrm{C}$ for 3 days, the MIC readings between control (without any hydrogen peroxide) and $\mathrm{H}_{2} \mathrm{O}_{2}(200 \mathrm{mM})$ treatments were compared by measuring OD 600 with Perkin Elmer Ensight. Decreased MIC values in the presence of hydrogen peroxide indicate that the chemicals have antifungal activity which specifically inhibits ergosterol pathway. The MIC assays were performed in triplicate.

\subsection{Cell membrane ergosterol quantification}

The fungal culture conditions for sterol extraction were performed with the methods described by Pinto with minor changes [21]. In brief, cell suspensions ( $200 \mu \mathrm{L}, 1 \times 10^{4}$ spores $\left./ \mathrm{mL}\right)$ were inoculated in $20 \mathrm{~mL}$ of medium with chelerythrine and sanguinarine, together with miconazole as a positive control and DMSO aqueous solution as a negative control. The culture tubes were placed at $28^{\circ} \mathrm{C}$ for 3 days shaking at $200 \mathrm{rpm}$. Fungal hyphae were then collected by centrifugation at $3000 \mathrm{rcf}$ for 10 minutes, washed three times with phosphate buffered saline (PBS), dried by lyophilization and weighted. The same weight of dried hyphae in different treatments was separately transferred to glass tubes contained $15 \mathrm{~mL}$ of $10 \%$ alcoholic $\mathrm{KOH}$ solution, followed by vigorous vortical agitation. The mixture was placed into the $80^{\circ} \mathrm{C}$ water bath for 90 minutes. As tubes cooled, sterols were extracted with petroleum ether (boiling range $30-60^{\circ} \mathrm{C}$ ). The free sterols in the phase of petroleum ether were transferred to clean glass tubes and heated to dry. The dried sterols were subsequently dissolved into $1 \mathrm{~mL}$ of methanol and analyzed by high performance liquid chromatography (HPLC) [22]. In brief, the chromatography was carried out with an Agilent 1100 HPLC system coupled with a DAD detector (Agilent technologies, Santa Clara, CA, USA). A C18 column $(3.5 \mu \mathrm{m}, 3 \mathrm{~mm} \times 150$ $\mathrm{mm}$ ) was used for the ergosterol analysis. The mobile phase was $100 \%$ of HPLC grade methanol (Merck, Waltham, MA, USA), and the flow rate was at $0.4 \mathrm{~mL} / \mathrm{min}$ with an injection volume of $10 \mu \mathrm{L}$. The fungal ergosterol content was quantified by the absorbance recorded in the chromatograms, and comparison with external ergosterol standard at $282 \mathrm{~nm}$. 


\subsection{In silico docking}

Molecular docking analysis to study binding affinity of compounds was performed using AutoDock Vina 1.1.1 (The Scripps Research Institute, CA, USA). The CYP51 Ca (Lanosterol 14-alpha demethylase, $C$. albicans) protein was taken from the PDB database (PDB code: 5FSA). Docking grid was set to 75,75 and 70 points in $x, y$ and $z$ directions respectively, enclosing the active site of posaconazole. The complex with the minimum binding energy and the binding modes were illustrated by PyMOL Molecular Graphics System Version 1.3 (Schrödinger, LLC, New York, NY, USA).

\subsection{In vivo assessment}

Female guinea pigs (weight, $250 \mathrm{~g}$ ) were used for the in vivo antifungal study, which was approved by the laboratory animal ethics committee of Shenzhen University, China [Approval number: SYXK (Yue) 2014-0140]. Animals were randomly divided into nine groups with six animals in each group, and were placed in the air-conditioned room at $24^{\circ} \mathrm{C}$. Infections caused by dermatophytes were established by a minor revision of the method described by Ghannoum et al. $[23,24]$. In brief, animals were anaesthetized by intraperitoneal injection of chloral hydrate. Two separated areas of the back $\left(2.5 \mathrm{~cm} \times 2.5 \mathrm{~cm}, 6.25 \mathrm{~cm}^{2}\right)$ of the guinea pigs were shaved and dehaired, and were gently abraded using sterile 400-grit sandpaper. $100 \mu \mathrm{L}$ of conidia suspension $\left(1 \times 10^{8}\right.$ spores $/ \mathrm{mL}$ ) was then dabbed on the abraded skin. All animals affected exhibited a range of typical clinical signs on day 10 after infection. A KOH microscopic examination of skin scraping also revealed hyphae and spores. Topical treatments with chelerythrine and sanguinarine were then applied daily to the infected area on day 11 of post-infection. The topical treatments were continued for 10 days. Analyses consisted of a clinical evaluation [25] and subsequent mycological evaluation were used to determine the status of dermatophytosis model by direct microscopic examination of scrapings from a $\mathrm{KOH}$ preparation site revealing hyphae [26,27].

\subsection{Clinical assessment of the lesions}

Clinical assessment of each animal is acquired with a semi-quantitative score according to the clinical signs in comparison with negative group based on the previous methods [24]. The clinical signs in the infected skin of animals in each group were recorded. The scores were calculated to evaluate the therapeutic efficacy.

\subsection{Pathological examination}

Skin biopsy specimens were collected under anesthesia from the guinea pigs inoculated with $T$. rubrum after 10 days of treatment, and were immediately fixed in Carnoy's solution within 24 hours. The tissues were sectioned at $5 \mu \mathrm{m}$ thickness and stained with Periodic Acid-Schiff (PAS) for histologic observation by light microscope.

\subsection{Statistical analysis}

All experiments that were performed in triplicate had statistical analysis performed using a t-test with GraphPad Prism 5.0 software for Windows, GraphPad Software, La Jolla California USA, www.graphpad.com. P-values of $<0.05$ were considered statistically significant. 


\section{Results}

\subsection{Hydrogen peroxide reduces fungal resistance in the presence of antifungal drugs}

In order to validate the introduction of an oxidizing agent as a drug discovery screening tool, MIC with addition of GSH, hydrogen peroxide, or sorbitol was performed (Table 1). The change of the MIC values was calculated by comparison of the control to GSH, hydrogen peroxide, or sorbitol (Figure 1a \& 1b). The addition of sorbitol is used to replicate previous research findings and was used here as a negative control [11,12]. The pathogenic fungus, T. rubrum, tended to be more robust and require higher concentrations of antifungal agents for growth inhibition. However, this trend was reversed for amorolfine. In the M. gypseum MIC assay, a decrease in drug resistance was observed in the presence of hydrogen peroxide in six out of ten drugs tested. On the contrary, GSH strongly protected T. rubrum against antifungal drugs classes, such as azoles and allylamines. However, its drug resistance in the presence of hydrogen peroxide was reduced across different classes of drug, such as ITZ, TCZ (two azole compounds), AB (competitive ergosterol), GF (cell division), and CF (enzyme inhibition). This indicated that hydrogen peroxide reduced resistance to many classes of antifungal drugs in both T. rubrum and M. gypseum, but the modes of inhibition/protection are different between the two species.

Table 1. A set of MIC values in four different conditions. The bolded number represents the value is higher than the control while the underlined number indicate the MIC value is lower when comparing to the control.

\begin{tabular}{|c|c|c|c|c|c|c|c|c|}
\hline Fungus & \multicolumn{4}{|c|}{ M. gypseum MIC $(\mathrm{ng} / \mathrm{mL})$} & \multicolumn{4}{|c|}{ T. rubrum MIC (ng/mL) } \\
\hline Drug & Control & 0.8M Sorbitol & $0.5 \mathrm{~g} / \mathrm{L} \mathrm{GSH}$ & $200 \mathrm{mM} \mathrm{H}_{2} \mathrm{O}_{2}$ & Control & 0.8M Sorbitol & $0.5 \mathrm{~g} / \mathrm{L} \mathrm{GSH}$ & $200 \mathrm{mM} \mathrm{H}_{2} \mathrm{O}_{2}$ \\
\hline BFZ & 400 & 400 & 800 & $\underline{200}$ & 3,200 & 12,800 & 51,200 & 6,400 \\
\hline ITZ & 800 & 800 & 800 & 200 & 800 & 800 & 800 & $\underline{200}$ \\
\hline MCZ & 160 & 160 & 320 & 160 & 320 & 320 & 1,280 & 320 \\
\hline TCZ & 80 & 80 & 320 & 80 & 320 & 320 & 1,280 & $\underline{160}$ \\
\hline $\mathrm{AF}$ & 160 & 160 & 320 & $\underline{80}$ & 20 & 20 & 80 & 20 \\
\hline $\mathrm{BF}$ & 64 & $\underline{32}$ & 64 & $\underline{16}$ & 1,600 & 3,200 & 12,800 & 1,600 \\
\hline $\mathrm{TF}$ & 8 & 8 & 16 & $\underline{4}$ & 25,600 & 25,600 & 102,400 & 51,200 \\
\hline $\mathrm{AB}$ & 3,200 & 3,200 & 6,400 & 3,200 & 3,200 & $\underline{800}$ & 3,200 & 3,200 \\
\hline GF & 12,800 & 12,800 & 12,800 & 12,800 & 102,400 & 102,400 & 102,400 & $\underline{51,200}$ \\
\hline $\mathrm{CF}$ & 1,600 & 1,600 & 1,600 & 1,600 & 1,600 & 1,600 & 1,600 & $\underline{400}$ \\
\hline
\end{tabular}
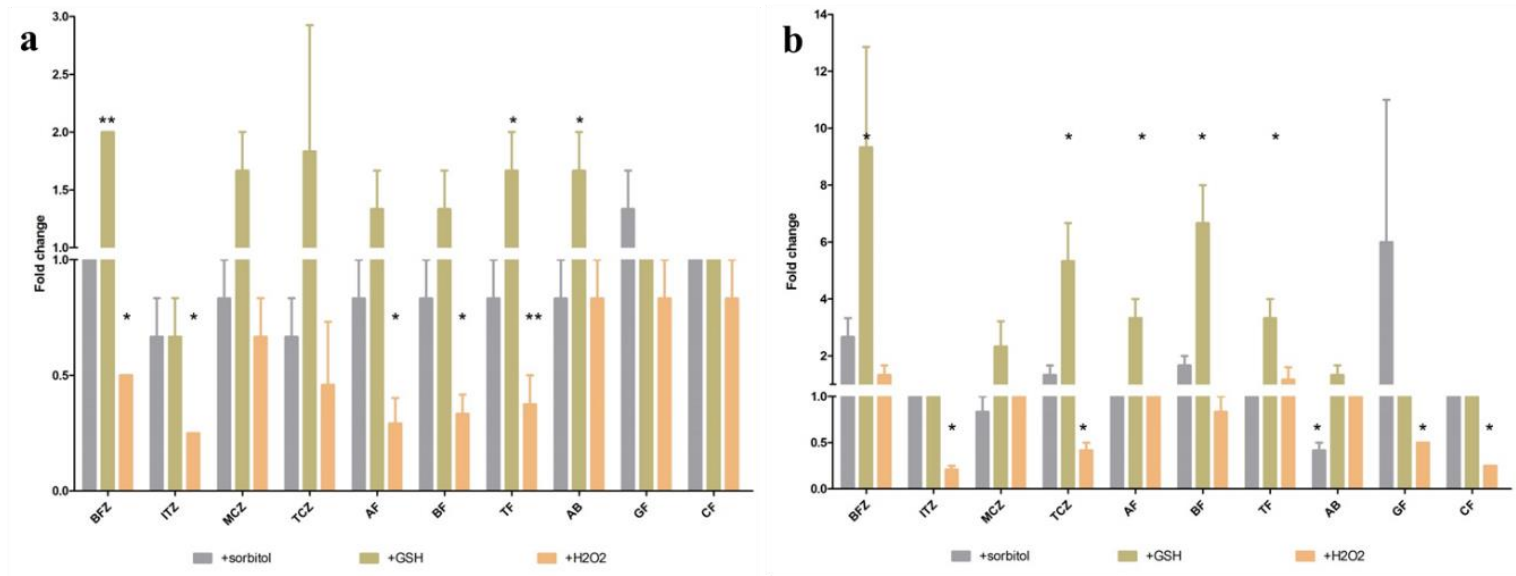

Figure 1. The fold change of MIC values comparing to control in addition of reagent to protect or induce oxidative stress (a) in M. gypseum (b) in T. rubrum. Fold change at 1.0 indicates no change between the control and sample. Asterisk $\left(^{*}\right)$ represents $p<0.05$ while $\left.{ }^{* *}\right)$ has $p$ value of $<0.01$. 


\subsection{Hydrogen peroxide induces fungal death}

Calcofluor white has been established as a marker for fungal chitin which is especially applicable for detecting the integrity of the cell walls in fungi [28,29]. This methodology was used to identify fungi treated with anti-ergosterol synthesis drugs in the presence of hydrogen peroxide. The Calcofluor white-stained fungi displayed similar fluorescence intensity and morphology when treated with all ten drugs even in the presence of hydrogen peroxide, suggesting that the cell wall is not compromised by the presence of hydrogen peroxide (Figure 2a-d and i).
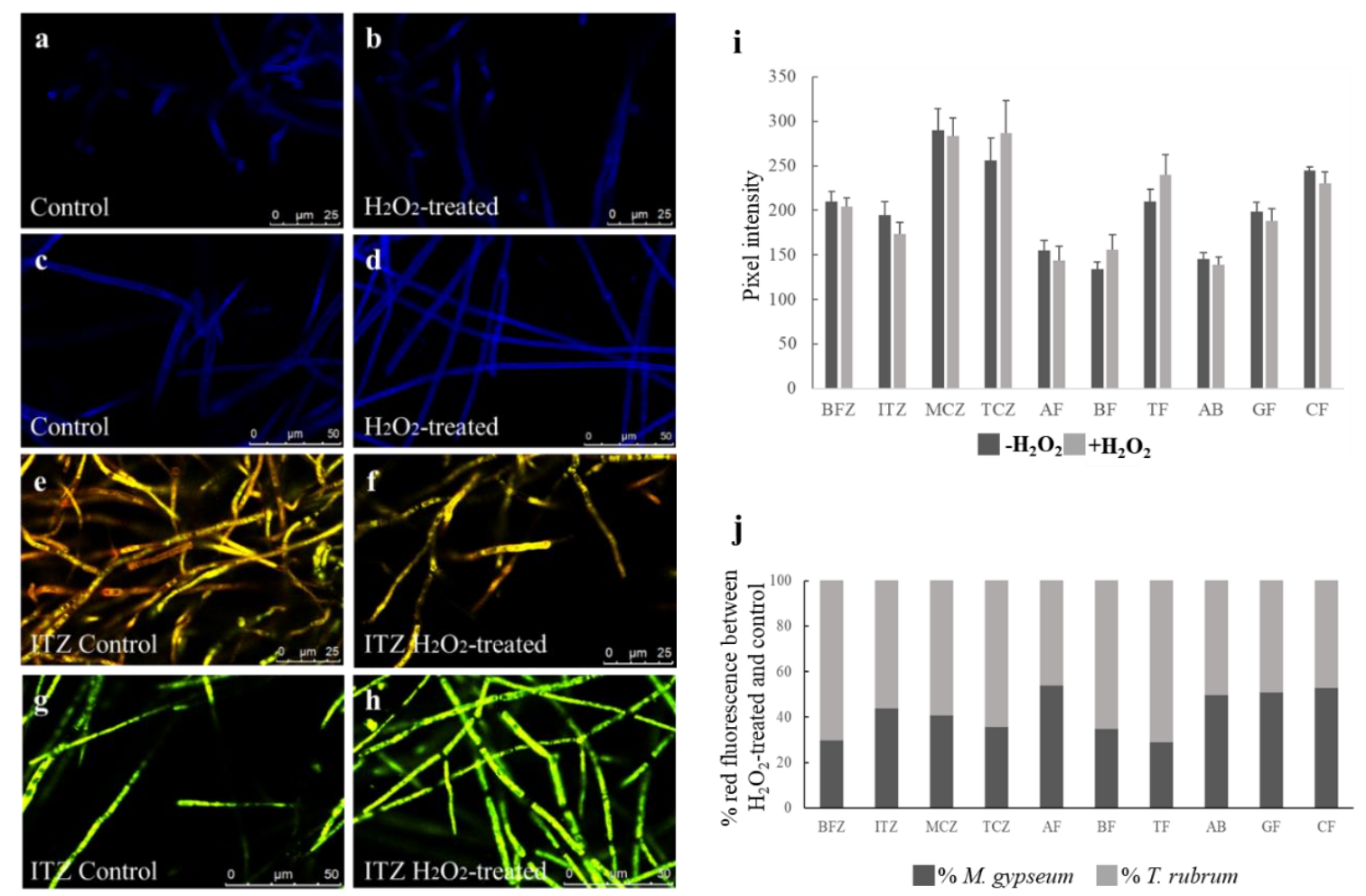

Figure 2. Confocal images of fungi treated with or without hydrogen peroxide and then stained with $(\mathrm{a}, \mathrm{b})$ Calcofluor white in M. gypseum, $(\mathrm{c}, \mathrm{d})$ Calcofluor white in T. rubrum, (e,f) Fun-1 stain in $M$. gypseum, and $(\mathrm{g}, \mathrm{h})$ Fun-1 stain in T. rubrum. (i)The blue pixel intensity of Calcofluor white in the presence and absence of hydrogen peroxide in M. gypseum. (j) The relative percentage of red fluorescence of Fun-1 stain is compared between hydrogen peroxide-treated and control in both $M$. gypseum and T. rubrum.

Fun-1 stain was used to determine fungal viability by observing the emission of the two-color fluorescence probe. Red fluorescence marks the intravacuolar structures of metabolically active cells and requires plasma membrane integrity, while dead cells emit green fluorescence due to the lack of metabolic activity in the intravacuolar bodies. It was observed that M. gypseum had a higher percentage of green fluorescent cells than red fluorescent cells in the presence of hydrogen peroxide with azole/allylamine drugs (Figure $2 \mathrm{e}-\mathrm{f}$ and $\mathrm{j}$ ), indicating hydrogen peroxide induced more cell death on fungi with cell wall membrane deficiency. Intriguingly, the degree of red and green fluorescence was not significantly different between the presence and absence of hydrogen peroxide treatments across all ten drugs spectrum in T. rubrum (Figure $2 \mathrm{~g}$-h and $\mathrm{j}$ ).

\subsection{Hydrogen peroxide influences the development of mycelia}

To understand the mode of inhibition of hydrogen peroxide in fungi, mycelial development in the presence of hydrogen peroxide was observed. In Figure 3a-d, M. gypseum germinated differently from that of T. rubrum. After 48 hours, T. rubrum had a larger average hyphal mass than M. gypseum (Figure 3e). A majority of M. gypseum spores germinated (54\%) and developed into short mycelia while less than $10 \%$ of T. rubrum spores germinated in the presence of hydrogen peroxide. The 
germinated T. rubrum developed longer and more branched out mycelia that would turn into masses of hypha by 24 hours.
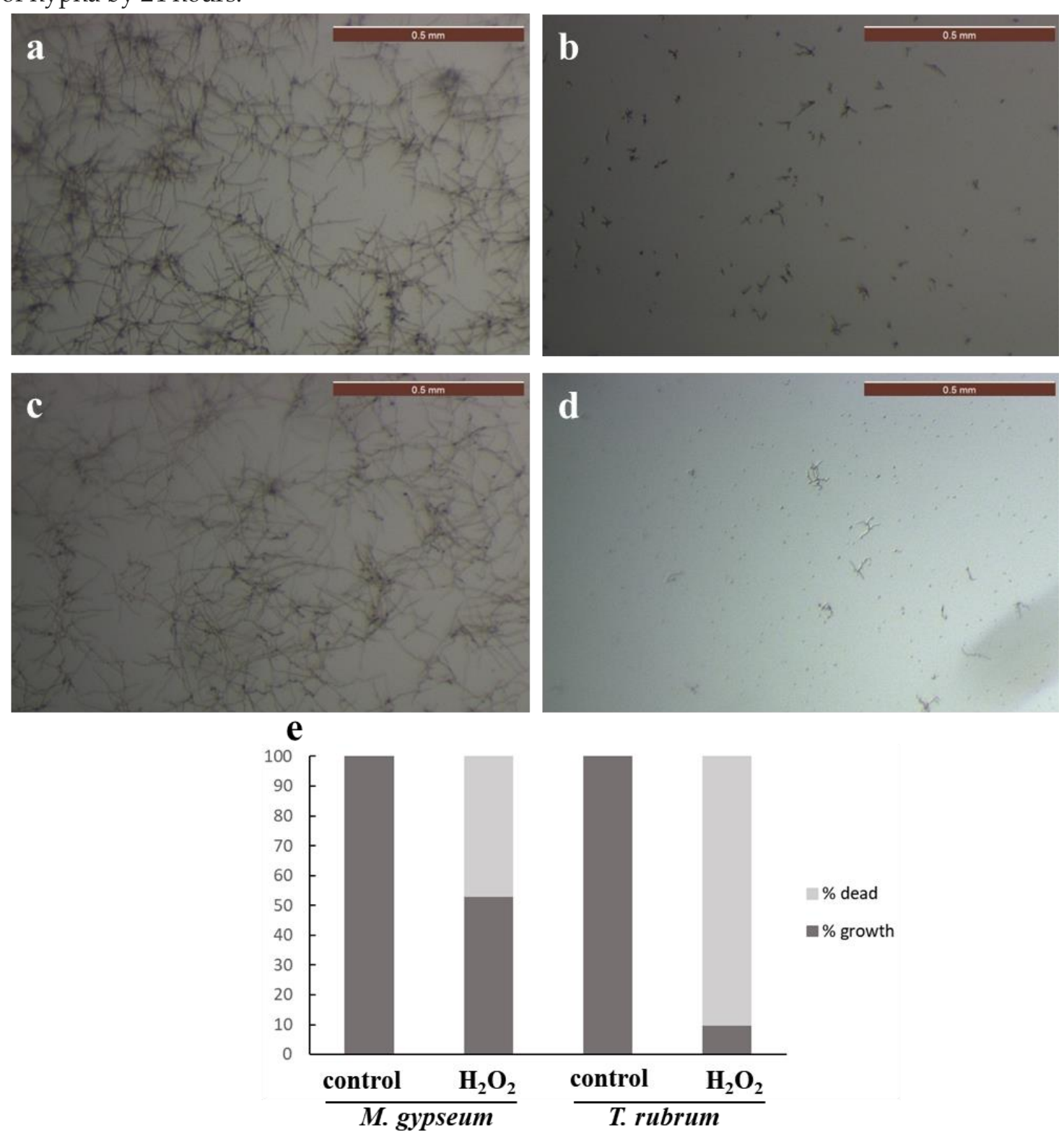

Figure 3. The physiological growth of mycelia (a)M. gypseum in control condition, (b) M. gypseum in hydrogen peroxide condition, (c) T. rubrum in control condition, and (d) T. rubrum in hydrogen peroxide condition. (e) The percentage mycelia growth of T. rubrum and M. gypseum in the presence and absence of hydrogen peroxide.

\subsection{Interactions of hydrogen peroxide with ergosterol, lanosterol, and squalene}

First, an Amplex Red control experiment was performed to show that ergosterol, squalene, lanosterol, and solvents do not interact with Amplex Red reagents. The addition of squalene in the presence of hydrogen peroxide did not significantly change the concentration of hydrogen peroxide (Table 2 and Figure 4). However, when lanosterol and ergosterol were mixed with hydrogen peroxide, a significant reduction of hydrogen peroxide between 7 to $14 \%$ was observed. 
Table 2. A summary of amount of normalized hydrogen peroxide remained in the presence of ergosterol, lanosterol, or squalene and their percentage changes in the presence of $10 \mu \mathrm{M}$ hydrogen peroxide. Blank represents the detection of Amplex Red reagent in the presence of dissolving solvent. Asterisk $\left(^{*}\right)$ represents $\mathrm{p}<0.05$ while $\left(^{* *}\right)$ has $\mathrm{p}$ value of $<0.01$

\begin{tabular}{|c|c|c|c|c|}
\hline Normalized $\left[\mathrm{H}_{2} \mathbf{O}_{2}\right]$ & Ergosterol & Lanosterol & Squalene & Blank \\
\hline$-\mathrm{H}_{\mathbf{2}} \mathbf{O}_{\mathbf{2}}(\mu \mathrm{M})$ & 0.13 & 0.15 & 0.15 & 0.15 \\
\hline $\mathbf{+ H}_{\mathbf{2}} \mathbf{O}_{\mathbf{2}}(\mu \mathrm{M})$ & $7.79^{* *} \pm 0.37$ & $8.46^{*} \pm 0.22$ & $8.64 \pm 0.06$ & $9.04 \pm 0.37$ \\
\hline$\%$ different from blank & $14 \%$ & $7 \%$ & $4 \%$ & --- \\
\hline
\end{tabular}
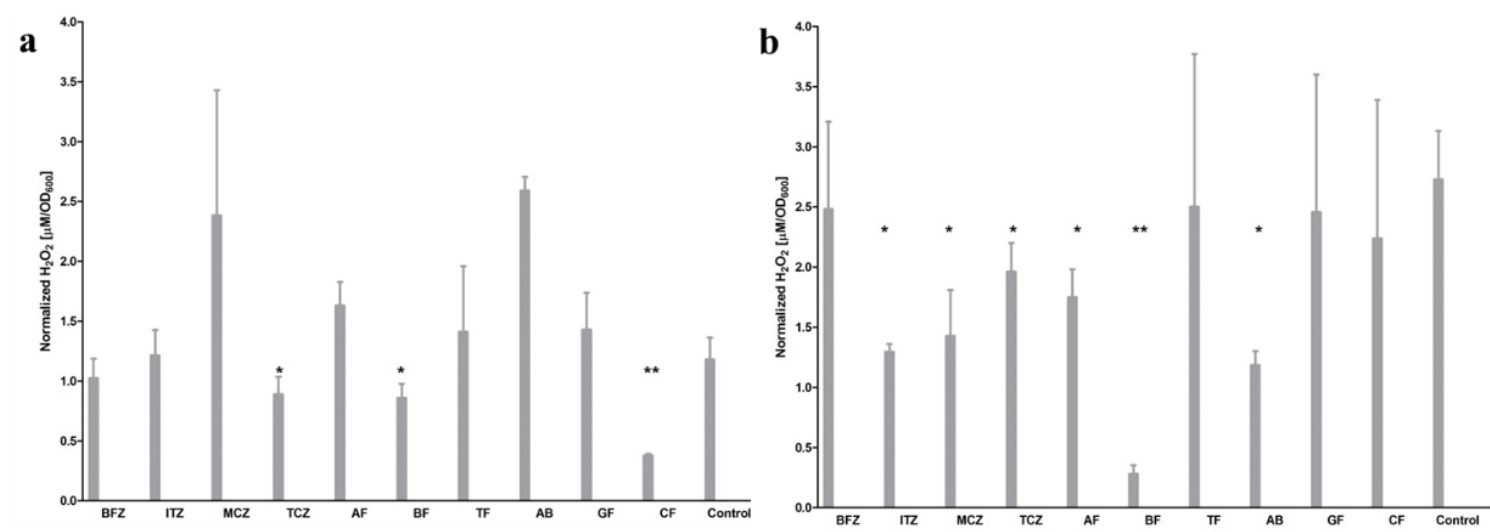

Figure 4. The normalized hydrogen peroxide detected by Amplex Red in each assay of (a) T. rubrum (b) M. gypseum. The values of each antifungal drug were compared with that of the control. Asterisk ${ }^{*}$ ) represents $\mathrm{p}<0.05$ while $\left(^{* *}\right)$ has $p$-value of $<0.01$.

\subsection{Ergosterol detection}

Ergosterol (tr 6.2-6.9 min) was detected at its maximum absorption wavelength $(282 \mathrm{~nm})$, and it was identified by the external standard. Both chelerythrine and sanguinarine are active against $T$. rubrum, and miconazole is known as an inhibitor of ergosterol biosynthesis. A representitive HPLC analysis is shown in Figure 5 Ergosterol levels in T. rubrum were significantly lower in the chelerythrine and sanguinarine groups $(8 \mu \mathrm{M})$ in comparison with control, and $4 \mu \mathrm{M}$ miconazole also exhibited lower ergosterol levels than that of the control. T. rubrum was more sensitive to sanguinarine than to chelerythrine, as indicated by the lower ergosterol contents in the treatment of sanguinarine group, which is consistent with the in vitro antifungal ability of the two compounds.

$1,3-\beta$-Glucan is a main structural component in cell wall of fungus. Quantification of $1,3-\beta$-glucan content was performed by fluorometric aniline blue staining method [30, 31]. No changes were found in the measurable $1,3-\beta$-glucans in T. rubrum after treatment with chelerythrine and sanguinarine (Data are not shown here). 

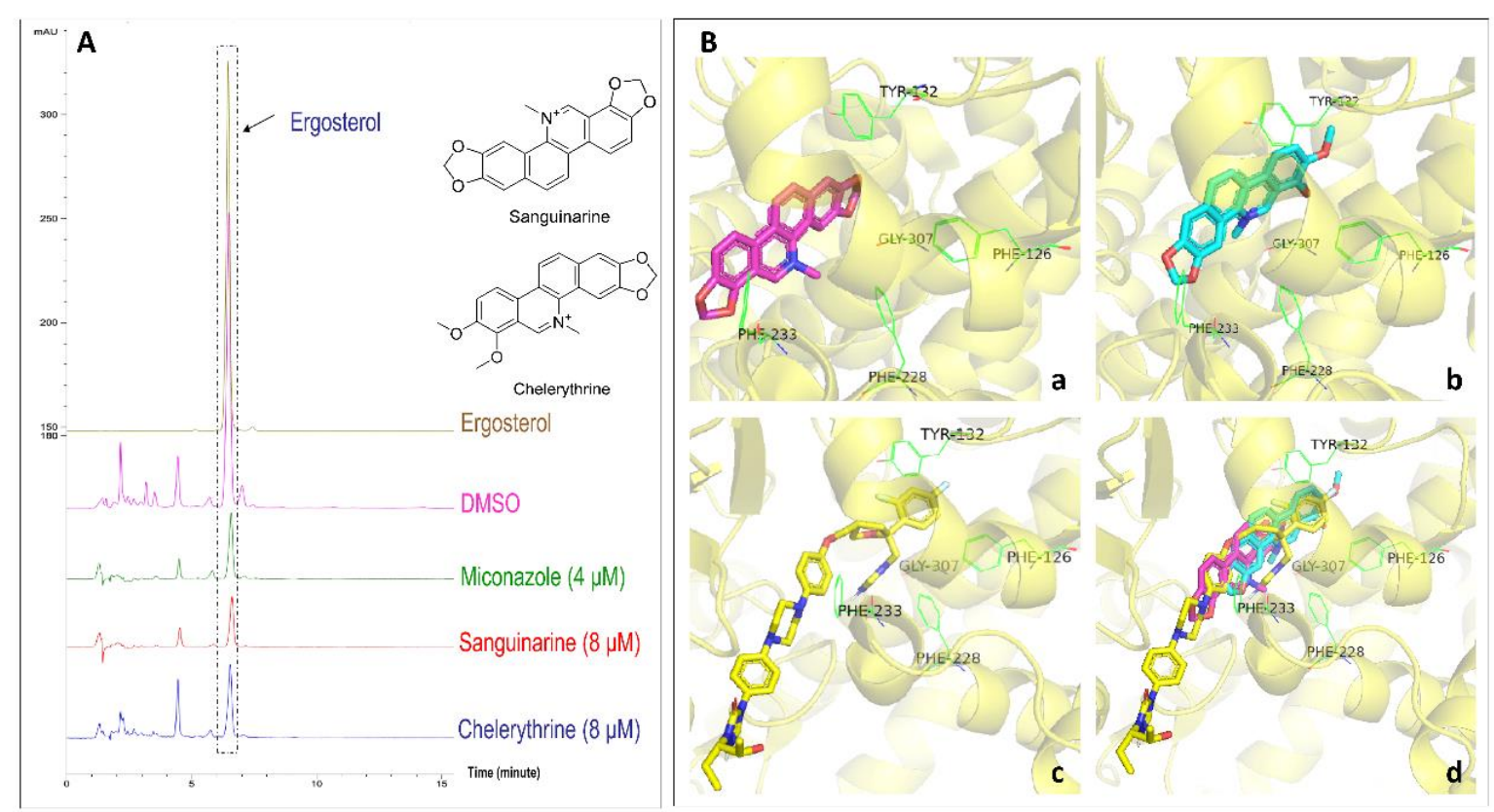

Figure 5. A. HPLC chromatogram of ergosterol obtained from the extracts of hyphae of T. rubrum treated with miconazole, chelerythrine and sanguinarine. B. In docking of sanguinarine and chelerythrine binding to CYP51 Ca (C. albicans). a, b and c. Stereoscopic view of CYP51 Ca with sanguinarine (a), chelerythrine (b) and itraconazole (c) bound in active site. (d) Overlap binding view of sanguinarine and chelerythrine with intraconzazole.

\subsection{MIC screen for detecting antifungal activity specific to the ergosterol pathway}

Chelerythrine and sanguinarine in our natural products bank (Figure 5) were found to reduce ergosterol biosynthesis in the HPLC analysis. We have shown that the addition of $200 \mu \mathrm{M}$ hydrogen peroxide in MIC assay using standard antifungal drugs resulted in significant reduction in MIC values (increased potency, Figure 1), especially for the classes of drugs that inhibits ergosterol biosynthesis. By using this modified MIC assay, both chelerythrine and sanguinarine were found to be four folds more potent against $M$. gypseum in the presence of hydrogen peroxide when compared to the control treatment (Table 3). The relative antifungal activity of both compounds is consistent with the modified MIC assay and the HPLC analysis further validates the integrity of this novel screening method.

Table 3. Antifungal activity of chelerythrine and sanguinarine against M. gypseum in control and hydrogen peroxide MIC conditions.

\begin{tabular}{llll}
\hline MIC reading & Control $(\mu \mathrm{M})$ & $\mathrm{H}_{2} \mathrm{O}_{2}(\mu \mathrm{M})$ & Fold change \\
\hline Chelerythrine & 100 & 25 & 4 \\
Sanguinarine & 50 & 12.5 & 4 \\
\hline
\end{tabular}

\subsection{Docking analysis}

Docking studies showed that both sanguinarine and chelerythrine were bound very close to the active site pocket of CYP51 Ca. The binding affinities of both sanguinarine and chelerythrine to CYP51 Ca are predicted to be similar with posaconazole. The residues around sanguinarine and chelerythrine are predominantly hydrophobic (Phe126 and Phe228). In our model the distal phenyl ring with methoxyls in the chlerythrine has aromatic contacts with Tyr132, and there is a hydrogen bond between the nitrogen and Gly307 hydroxyl. While five-membered two oxygens containing heterocycles in sanguinarine interact with residue Phe233 and Tyr132. The residue Phe233, also interact with five-membered heteroaromatic rings with two oxygens in chelerythrine. 
3.8. Efficacy of once-daily administration of chelerythrine and sanguinarine in a guinea pig model of dermatophytosis

The comparison of the appearance of the infected area among groups with different degrees of skin lesions is shown in Figure 6. The animals typically showed skin lesions after ten days of infection. Skin lesion scores decreased after treatment with miconazole, and all dose groups of chelerythrine and sanguinarine. Statistically significant differences in the lesion scores were observed among the vehicle group and the groups receiving miconazole and all doses of chelerythrine and sanguinarine $(P<0.001)$. Table 4 shows that the lesion scores quickly declined in the groups of the low dosage of chelerythrine $\left(0.1 \mathrm{mg} / \mathrm{cm}^{2}, 0.55 \pm 0.16 ; P<0.05\right)$, middle dosage of chelerythrine $\left(0.2 \mathrm{mg} / \mathrm{cm}^{2}, 0.5 \pm 0.1\right.$; $P<0.05)$ and high dosage of chelerythrine $\left(0.4 \mathrm{mg} / \mathrm{cm}^{2}, 0.5 \pm 0.16 ; P<0.05\right)$ compared with the model group. Similarly, lesion scores were significantly different with that of the model group $(P<0.05)$ among the different dosage groups of sanguinarine. The lesion scores in low $\left(0.1 \mathrm{mg} / \mathrm{cm}^{2}, 0.55 \pm 0.14\right.$; $P<0.05)$, middle $(0.5 \pm 0.09 ; P<0.05)$ and high dosages of sanguinarine $(0.5 \pm 0.1 ; P<0.05)$ were considerably lower than that of model group. Both chelerythrine and sanguinarine groups showed the similar lesion scores with miconazole group. In addition, the chelerythrine and sanguinarine groups demonstrated higher clinical efficacies (ranging from $89 \%$ to $94 \%$ ) than the model group $(P<$ $0.001)$.

Table 4. Therapeutic effect of chelerythrine and sanguinarine in a guinea pig model of dermatophytosis ${ }^{a}$

\begin{tabular}{|c|c|c|c|c|c|}
\hline \multicolumn{2}{|l|}{ Treatment } & $\begin{array}{l}\text { Administrat } \\
\text { ion period } \\
\text { (days after } \\
\text { infection) }\end{array}$ & $\begin{array}{l}\text { Number } \\
\text { of } \\
\text { infection } \\
\text { site }\end{array}$ & $\begin{array}{l}\text { Score of lesion } \\
\text { on the infection } \\
\text { site }\end{array}$ & $\begin{array}{l}\text { Clinical } \\
\text { efficacy (\%) }\end{array}$ \\
\hline \multicolumn{2}{|l|}{ Negative control $^{b}$} & & & $\mathrm{NA}^{d}$ & NA \\
\hline \multicolumn{2}{|l|}{ Model group ${ }^{c}$} & & & $7.6 \pm 0.33$ & NA \\
\hline \multicolumn{2}{|l|}{ Miconazole } & & & $0.5 \pm 0.13^{*_{e}}$ & $93.42 \pm 1.66$ \\
\hline \multirow[t]{3}{*}{ Chelerythrine } & $\begin{array}{l}\text { Low } \\
\left(0.1 \mathrm{mg} / \mathrm{cm}^{2} / \mathrm{d}\right)\end{array}$ & & & $0.55 \pm 0.16^{*}$ & $89.47 \pm 2.16$ \\
\hline & $\begin{array}{l}\text { Middle } \\
\left(0.2 \mathrm{mg} / \mathrm{cm}^{2} / \mathrm{d}\right)\end{array}$ & & & $0.5 \pm 0.1^{*}$ & $93.41 \pm 1.38$ \\
\hline & $\begin{array}{l}\text { High } \\
\left(0.4 \mathrm{mg} / \mathrm{cm}^{2} / \mathrm{d}\right)\end{array}$ & 10 & 12 & $0.5 \pm 0.16^{*}$ & $93.42 \pm 2.20$ \\
\hline \multirow[t]{3}{*}{ Sanguinarine } & $\begin{array}{l}\text { Low } \\
\left(0.1 \mathrm{mg} / \mathrm{cm}^{2} / \mathrm{d}\right)\end{array}$ & & & $0.55 \pm 0.14^{*}$ & $90.79 \pm 1.81$ \\
\hline & $\begin{array}{l}\text { Middle } \\
\left(0.2 \mathrm{mg} / \mathrm{cm}^{2} / \mathrm{d}\right)\end{array}$ & & & $0.5 \pm 0.09^{*}$ & $93.42 \pm 1.17$ \\
\hline & $\begin{array}{l}\text { High } \\
\left(0.4 \mathrm{mg} / \mathrm{cm}^{2} / \mathrm{d}\right)\end{array}$ & & & $0.5 \pm 0.1^{*}$ & $93.43 \pm 1.16$ \\
\hline
\end{tabular}

${ }^{a}$ Guinea pigs were randomly divided into 9 groups, with 6 animals in each group.

${ }^{b}$ Guinea pigs in negative control group were uninfected and received saline.

cAnimals in model group were infected and received saline.

${ }^{d} \mathrm{NA}$, not applicable.

${ }^{*} P<0.05$ versus model group.

The animals in the model group exhibited patchy hair loss, abundant skin scales and visible skin ulcer (Figure 6b). In contrast, animals receiving miconazole showed new hair growth, with no obvious signs of infection such as redness, swelling and scarring (Figure 6c). Animals in groups of chelerythrine and sanguinarine $\left(0.1,0.2\right.$ and $\left.0.4 \mathrm{mg} / \mathrm{cm}^{2}\right)$ also exhibited healthy and smooth skin without clinical infection signs (Figure 6d-i). These symptomatological findings indicate that both chelerythrine and sanguinarine were effective in treating dermatophyte infection in animal models at a very low dose $\left(0.1 \mathrm{mg} / \mathrm{cm}^{2}\right)$. 

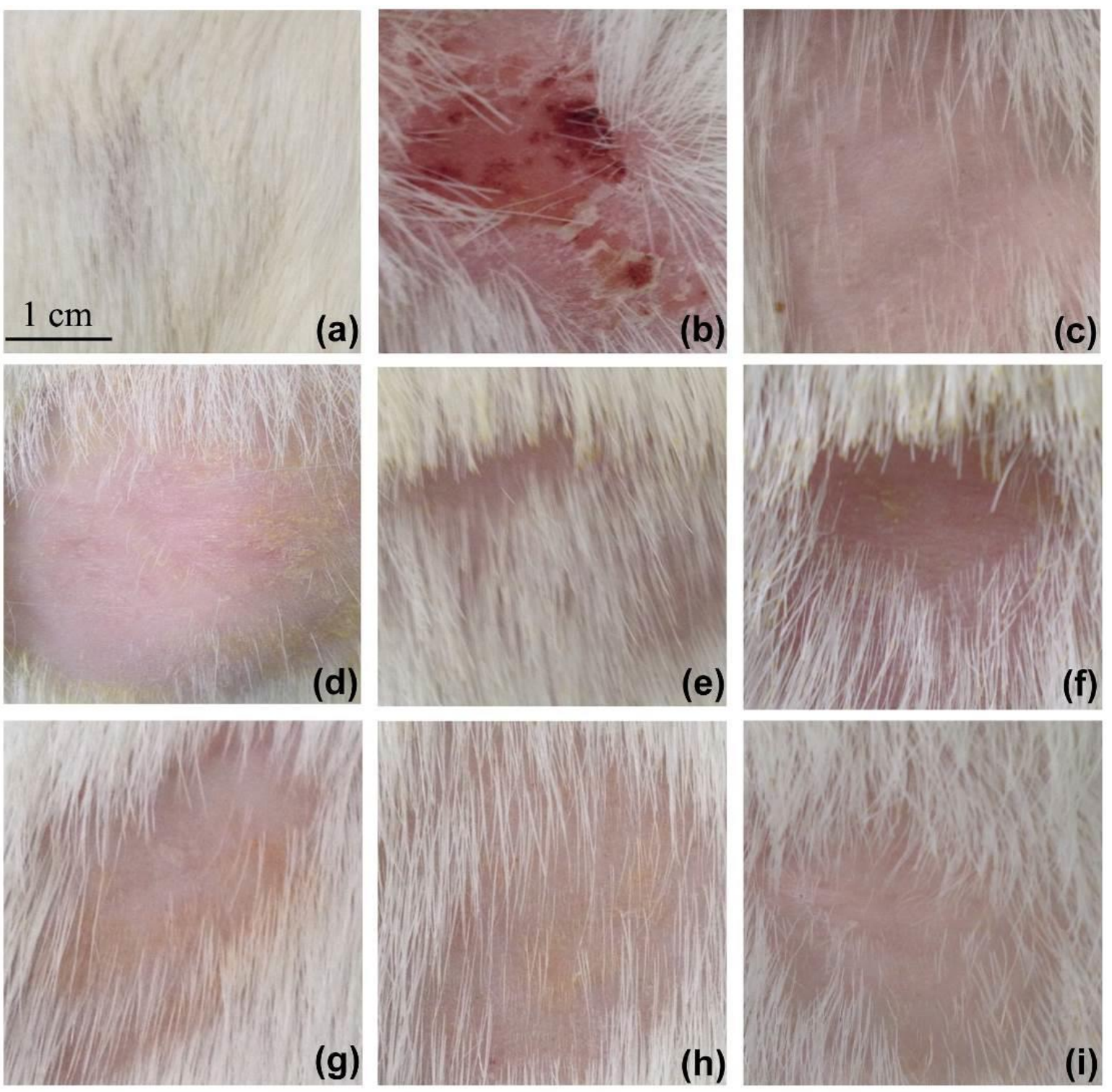

Figure 6. Appearances of the inoculation sites of the guinea pigs in different treatment groups. Groups of six guinea pigs were treated with once daily administration of miconazole $((\mathrm{c}), 0.2$ $\mathrm{mg} / \mathrm{cm}^{2} / \mathrm{d}$ ), chelerythrine (d)-(f) and sanguinarine (g-i) for 10 days. ((d) and (g), low concentration 0.1 $\mathrm{mg} / \mathrm{cm}^{2} / \mathrm{d}$; (e) and (h), middle concentration $0.2 \mathrm{mg} / \mathrm{cm}^{2} / \mathrm{d}$; (f) and (i), high concentration 0.4 $\mathrm{mg} / \mathrm{cm}^{2} / \mathrm{d}$ ), or vehicle (b) after infection, and normal control(a).

\subsection{Histopathological evaluation}

Animals in the negative control group presented normal histology of epidermis and dermis (Figure 7a), while the diseased model group showed severe epidermal proliferation with hyperkeratosis and retention of corneocytes accompanied with typical inflammatory infiltrates in dermis. Animals treated with miconazole presented low degrees of thickening and proliferation with mild inflammatory response in the epidermis. With treatment of $0.1,0.2$ and $0.4 \mathrm{mg} / \mathrm{cm}^{2}$ of chelerythrine (groups (d) to (f)), no obvious increased epidermal thickness and hyperkeratosis were found in the guinea pigs. Similarly, groups (g), (h) and (i) $\left(0.1,0.2\right.$ and $0.4 \mathrm{mg} / \mathrm{cm}^{2}$ of sanguinarine, respectively) showed little or no thickening of the stratum corneum.

We have also observed a strong PAS reaction in the basement membrane of the epithelium, hair follicles and sebaceous gland in the animals of the model group. Meanwhile, a weak PAS positive reaction was found in the infected skin treated with miconazole and all doses of chelerythrine and sanguinarine. However, hyphae or spores could rarely be visualized by PAS staining because of the exclusion by increased epidermal turnover and spontaneously healing [32]. The intensity of the 
positive reaction was decreased after 10 days of chelerythrine and sanguinarine treatment which is similar to that of the control group.
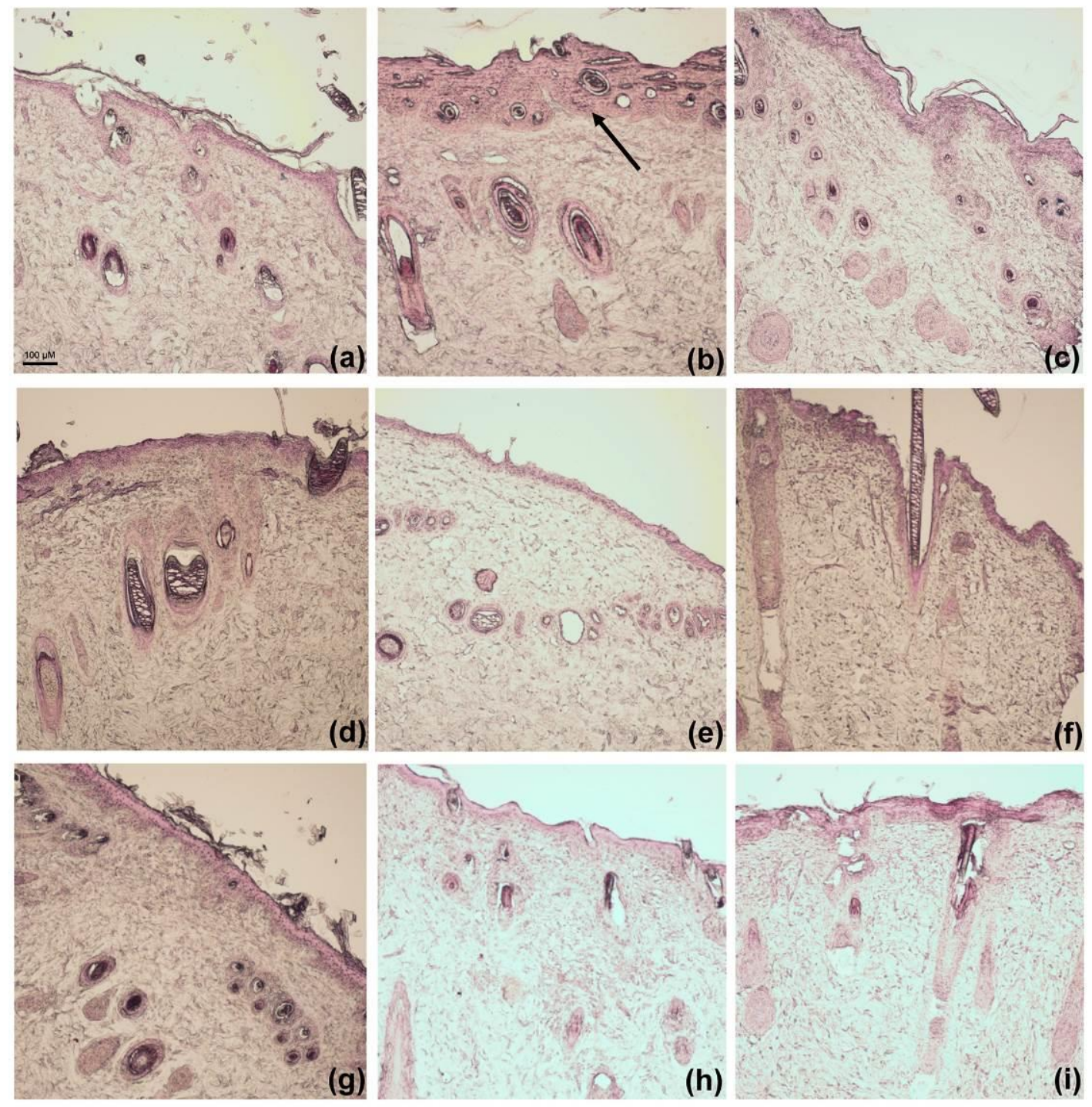

Figure 7. Skin tissues from guinea pigs under different treatments showing the inflammatory scale and the thickness of epidermis layer. PAS stain, 100X magification. (a) normal control; (b) model group treated with medium; (c) Miconazole, $0.2 \mathrm{mg} / \mathrm{cm}^{2} / \mathrm{d}$; (d)-(f) Chelerythrine, $0.1,0.2$ and 0.4 $\mathrm{mg} / \mathrm{cm}^{2} / \mathrm{d}$, respectively; (g)-(i) Sanguinarine, $0.1,0.2$ and $0.4 \mathrm{mg} / \mathrm{cm}^{2} / \mathrm{d}$, respectively. In the skin of the model group, the number of dermal cells, and hydropic alterations (arrows, b), increased. Moderate PAS positive reaction was observed in the back skin of treated group animals.

\section{Discussion}

Current approaches to investigate mechanism of the antifungal drugs, such as examination of the morphological change of fungi [12], measurement of ergosterol or chitin [33], quantification of the target mRNA, screening of sterol analogs using gas chromatography mass spectrometry (GC-MS) [34], or even study of the change of transcriptome [35] can be expensive, laborious, and time consuming. Therefore, it is important to develop a method that could help with early diagnosis and screening for wider range of antifungal products.

T. rubrum and M. gypseum are good candidates for this study. In molecular level, M. gypseum and T. rubrum contain the largest numbers of unique genes among the most abundant dermatophytes and M. gypseum does not contain any gene specific to animal-associated dermatophytes confirm that the two species are of great study of interests [32]. Moreover, even with lower infection cases by $M$. gypseum, it should not be ignored that its infection outbreaks among young children who share 
common media, e.g., sandboxes, have been reported in several countries [36]. Those children are often misdiagnosed for an extend period of time. We could utilize our method as a potential quick screening tool for fungal classification at pediatric clinics.

The production of ROS in cells through normal metabolic processes is inevitable. Therefore, fungi have adapted and developed pathways to regulate hydrogen peroxide level in nanomolar range [37]. However, hydrogen peroxide in micromolar range causes oxidative stress and eventually cell apoptosis. By utilizing its unique property, we developed a hydrogen peroxide MIC screening assay to understand how different classes of fungi response to that oxidative stress in the presence of different concentrations/classes of antifungal drugs. We found that M. gypseum is more susceptible to antifungal drugs in the presence of hydrogen peroxide than T. rubrum, especially for the classes of drugs such as azoles and allylamines, which inhibit the ergosterol biosynthesis pathway leading to accumulation of lanosterol and squalene (Figure 1) [4,7]. The low inhibitory response of T. rubrum on hydrogen peroxide is due to the production of endogenous catalase [38] which catalyzes hydrogen peroxide into water and oxygen and thus reduces oxidative stress in cells. In a comparative gene analysis, while most of the genes involved in ergosterol biosynthesis in dermatophytes are wellconserved, a subset of genes, ERG4 and ERG25, are duplicated in M. gypseum [39]. In Saccharomyces cerevisiae, ERG25 is activated at an alternate ergosterol biosynthesis pathway leading to the production of toxic fungistatic sterol when the canonical pathway is disrupted with antifungal drug such as azoles [40]. This implicates the oxidation stress by hydrogen peroxide could induce toxin production in M. gypseum in the presence of azoles. In addition, M. gypseum's unique combination of polysaccharide deacetylase type 1 with LysM domain could play a role on defense from other fungi and cell wall modification which is absent in T. rubrum [39]. Therefore, those gene duplications could increase the sensitivity of hydrogen peroxide in $M$. gypseum due to the modulated composition of the cell membrane. As a result, the susceptibility to antifungal drugs and oxidative stress of M. gypseum could be easily influenced [41].

GSH is an abundant low-molecular-weight thiol that plays a key role on responding to oxidative stress, the maintenance of mitochondrial and membrane integrity [42]. In response to oxidative stress, glutathione and catalase play an overlapping defensive roles on protection against hydrogen peroxide [43]. With those endogenous catalases presence in T. rubrum mentioned in Calderon \& Shennon's paper (1987), the addition of extracellular GSH and endogenous catalase on T. rubrum could provide even greater protection against antifungal drugs than on M. gypseum. Thus, it is not surprising to observe several folds of increase in MIC readings in T. rubrum in the presence of GSH.

In the Fun-1 staining result (Figure 2e-h \& j), it showed metabolically higher percentage of cell death of M. gypseum than of T. rubrum could be due to a higher rate of cell wall diffusion of hydrogen peroxide when the cell wall membrane is defective [44]. The membrane structure is known to be different among different species of fungi [45]. This reasoning is aligned with our observation in the MIC values in T. rubrum as it had higher drug tolerance in most of the drug classes than M. gypseum in the presence of hydrogen peroxide which is an indication that the cell wall permeability of hydrogen peroxide and drugs could be much slower in T. rubrum than in M. gypseum [46].

With our finding in mycelia growth pattern on T. rubrum, hydrogen peroxide dramatically inhibited M. gypseum germination (Figure 3). Ironically, M. gypseum has shown to have overall higher percentage of myceli growth than T. rubrum on an agar medium [47]. However, the branching mass of mycelia is much shorter in M. gypseum than in T. rubrum suggesting hydrogen peroxide has larger influence in inhibiting mycelia branching in M. gypseum. It is also possible that the small population of $T$. rubrum spores that were germinated could have higher resistant to hydrogen peroxide, thus, mycelia branching is much more pronounced in T. rubrum. These finding supports our observation in our cell apoptosis results where hydrogen peroxide induced metabolites' cell death in mycelia of M. gypseum but not in T. rubrum (Figure 2e-h). Therefore, T. rubrum can strive in growing into big volume of hyphae.

Different classes of antifungal drugs that target ergosterol pathway have shown to alternate cell walls and, therefore, could change its cellular response to oxidative stress, such as hydrogen peroxide. By utilizing Amplex Red assay that measures the presence of hydrogen peroxide in bioassay, all but one of the fungi added with azole and allylamine classes of drugs (lanosterolenriched) have shown to have significantly lower amount of hydrogen peroxide left when comparing 
to the control in M. gypseum (Figure $4 \mathrm{~b}$ ). This suggested that hydrogen peroxide interacts with the lanosterol-enriched cell walls more, and thus causes higher oxidative stress to M. gypseum, which leads to cell death. This Amplex Red observation is consistent with our trend found in the modified MIC assay. However, the trend is quite unclear with T. rubrum as TCZ, BF, and CF which are in different classes of drugs suggesting that different species react to hydrogen peroxide differently due to the difference in cell wall composition among fungi (Figure 4a). It could also be due to the diverse development stages of mycelia in T. rubrum (Figure 3c-d) where many spores remain dominant in the presence of hydrogen peroxide with low population of spores germinated into large branches of mycelia which could obstruct the overall results.

The putative mechanism of some antifungal alkaloids was targeted to the cell membrane [48]. However, not many natural alkaloids have been studied for their antifungal molecular targets. We have determined that the potencies of the natural alkaloids chelerythrine and sanguinarine in inhibiting fungal growth with this novel screening tool. This result is also consistent with the findings of Tian et al. that the M. cordata total alkaloids containing these two compounds had potent intrinsic antifungal activity against the five strains that cause dermatophytosis [49]. The structures of chelerythrine and sanguinarine are very similar, and it is possible that both spatial and quaternary ammonium structures of these two compounds contribute to the antimicrobial activity. Our data indicate that 7,8-dimethoxy ring of chelerythrine had little influence on the antifungal activity (data not shown). In addition, 1,3- $\beta$-glucan synthase is another cell membrane target of antifungal drugs. Our results obtained from the ergosterol detection (Figure 5) corroborate that the alkaloids inhibited ergosterol biosynthetic pathways [50]. Yet, they showed no inhibitory effects on the synthesis of 1,3$\beta$-glucan (data are not shown), which indicate that this novel screening tool is precise in detecting antifungal compounds specifically target ergosterol pathway.

The CYP51 is a cytochrome P450 enzyme which catalyses the oxidative removal of the 14- $\alpha$ methyl group from sterol precursor, lanosterol, in the ergosterol biosynthesis pathway. Inhibition of CYP51 leads to a depletion of ergosterol from cell membranes, resulting in the inhibition of fungal growth [51]. HPLC-based assay provided evidence that the synthesis of ergosterol is inhibited by sanguinarine and chelerythrine, which may cause disruption of the cell membrane and its leakage suggesting azole drug based effect on the fungal CYP51. The modes of binding of lead inhibitors sanguinarine and chelerythrine with modeled C. albicans like CYP51 were identified using molecular docking with Autodock Vina (Figure5B). Docking studies showed that both sanguinarine and chelerythrine were bound very close to the active site pocket of CYP51 interacting with active site residues with high binding affinities, which is highly similar with that of posaconazole. In the absence of the crystal structure of CYP51 from T. rubrum interacting with its natural substrate, the interaction with the modeled CYP51 provides a rationale for designing lead inhibitors based on the active site and optimization of the structure activity relationship.

Considering the in vitro antifungal activity against T. rubrum, it was hypothesized that chelerythrine and sanguinarine might have therapeutic effectiveness in guinea pig model of dermatophytosis on topical application. However, the skin irritation assessment is essential for ensuring the safety external use of these potential agents [52]. The present investigation indicated that these two alkaloids application onto the skin of nude mice showed no sign of irritation even with 10 consecutive days of dosage exposure. Thus, the compounds may have potential in the application of external use in dermatophytosis.

Guinea pigs are widely used as dermatophyte animal models for infection due to their outstanding characteristics such as good reproducibility, high susceptibility and easy handling [53]. The symptoms of infected guinea pigs are strikingly similar to humans and lead to a better understanding of human disease which may aid antifungal drug development [53,54]. In examining once-daily dosing regimens, external application of chelerythrine and sanguinarine was indeed highly effective in improving the clinical lesion signs by inhibiting fungal growth. Interestingly, both compounds showed clinical efficacies that were indistinguishable among the three different concentrations of dosage, indicating that the two compounds are highly effective in low dosage.

The histopathological study of the skin lesions revealed serious inflammation and local skin thickening. The absence PAS staining of hyphae in infected tissue was correlated to the exclusion by self-renew of epithelial cells, variable degrees of acanthosis or proliferation and keratinization in the 
infected skin could still be observed by PAS staining [53,54]. In the current study, histopathological examination displayed that chelerythrine and sanguinarine had equal treatment effectiveness with miconazole on instauration of the infected animal skin.

\section{Conclusions}

Here we found that oxidative stressors/protectors as additions to MIC assays could be used to screen for novel anti-fungal drugs targeting ergosterol biosynthesis pathway. It is the first time to show that hydrogen peroxide could be used in MIC for screening purposes. Based on this screening tool, two antifungal isoquinoline alkaloids were discovered that inhibited fungal ergosterol biosynthesis via inhibiting fungal CYP51. Furthermore, in the in vivo study, chelerythrine and sanguinarine were found to exhibit excellent therapeutic effects in an animal model of dermatophytosis upon topical application. The study indicated the reliability of this novel screening tool for antifungal drugs targeting the ergosterol biosynthesis pathway.

In order to understand if external oxidative/protective stresses influence the transcriptional profiles of T. rubrum and M. gypseum targeting the membrane transportation and ergosterol pathways, qRT-PCR could be performed. Hydrogen peroxide has shown to affect the gene expression in membrane transportation pathway. Those genes are shown to be COR1 and QCR7 [22]. A comparison of the gene expression significantly affected by the azole class of antifungal drugs within the ergosterol pathway, like ERG11, ERG10, ERG6, and HES1, could gain an insight on the difference in membrane composition and drug response between those two species. This could help us farther understand on how those difference mechanisms influences fungal sensitivity against antifungal drugs $[33,55]$.

Author Contributions: S.W. set up antifungal screening tool and writing - original draft; X.S. carried out animal experiments and writing- original draft. T.N. performed antifungal screening. X.L., J.Z. and Z.Q. isolated and provided the two isoquinoline analogues; S.D. reviewed and edited the manuscript; Z.H. and H.Z. Designed and supervised the studies, formal analysis, writing, review and editing.

Funding: This research was funded by Faculty Research Grant, Hong Kong Baptist University (FRG 2/17-18/103), the Hong Kong Baptist University (HKBU) Interdisciplinary Research Matching Scheme (RC-IRMS/15-16/02), NSFC funding 31670360, 81973293, U1702286 and Natural Science Foundation of SZU 860-000002110131.

Acknowledgments: We thank you for Mr. King Wai Chan for helping us with confocal and stereomicroscope imaging. We appreciate Dr. Ka-Man Lai's help in editing the manuscript.

Conflicts of Interest: The authors declare no conflict of interest.

$\begin{array}{ll}\text { Abbreviations } & \\ \text { AB } & \text { amphotericin B } \\ \text { AF } & \text { amorolfine } \\ \text { BF } & \text { butenafine } \\ \text { BFZ } & \text { bifonazole } \\ \text { GF } & \text { griseofulvin } \\ \text { CF } & \text { ciclopirox } \\ \text { CYP51 } & \text { sterol 14 } \alpha \text {-demethylase } \\ \text { GC-MS } & \text { chromatography mass spectrometry } \\ \text { GH } & \text { glucose-HEPES } \\ \text { GSH } & \text { glutathione } \\ \text { H2O2 } & \text { hydrogen peroxide } \\ \text { HPLC } & \text { high performance liquid chromatography } \\ \text { ITZ } & \text { itraconazole } \\ \text { MCZ } & \text { miconazole }\end{array}$




$\begin{array}{ll}\text { MIC } & \text { minimum inhibitory concentration } \\ \text { OD } & \text { optical density } \\ \text { PAS } & \text { Periodic Acid-Schiff } \\ \text { PBS } & \text { phosphate buffered saline } \\ \text { PDB } & \text { potato dextrose broth } \\ \text { ROS } & \text { reactive oxygen species } \\ \text { WHO } & \text { World Health Organization } \\ \text { TCZ } & \text { tebuconazole } \\ \text { TF } & \text { terbinafine }\end{array}$

\section{References}

1. Havlickova, B.; Czaika, V.A.; Friedrich, M. Epidemiological trends in skin mycoses worldwide. Mycoses 2008, 51, 2-15.

2. Almeida, S.R. Immunology of dermatophytosis. Mycopathologia 2008, 166, 277-283.

3. Degreef, H. Clinical forms of dermatophytosis (ringworm infection). Mycopathologia 2008 166, 257-265.

4. de Aguiar, P.; Maranhão, F.; Rossi, A.; Martinez-Rossi, N.M. Dermatófitos: interação patógeno-hospedeiro E resistência a antifúngicos. Anais Brasileiros de Dermatologia 2010,85,657-667.

5. Crawford, F.; Hollis, S. Topical treatments for fungal infections of the skin and nails of the foot. Cochrane Database Syst Rev 2007, 3,CD001434.

6. Kathiravan, M.K.; Salake, A.B.; Chothe, A.S.; Dudhe, P.B.; Watode, R.P.M.; Mukta, S.; Gadhwe S. The biology and chemistry of antifungal agents: a review. Bioorg Med Chem 2012, 20, 5678-5698.

7. Ghannoum, M.A.; Rice, L.B. Antifungal agents: mode of action, mechanisms of resistance, and correlation of these mechanisms with bacterial resistance. Clin Microbiol Rev 1999, 12, 501-517.

8. Dupont, S.; Lemetais, G.; Ferreira, T.; Cayot, P.; Gervais, P.; Beney, L. Ergosterol biosynthesis: a fungal pathway for life on land? Evolution 2012,66, 2961-2968.

9. Alistair, J.P.; Brown, A.; Hall, T. Dynamic fungal cell wall architecture in stress adaptation and immune evasion. Trends in microbiology 2018,26, 284-295.

10. Rodrigues, M.L. The multifunctional fungal ergosterol. MBio 2018, 9.

11. de Oliveira Pereira,F.; Mendes, J.M.; de Oliveira Lima, E. Investigation on mechanism of antifungal activity of eugenol against Trichophyton Rubrum. Med Mycol 2013,51, 507-513.

12. Escalante, A.; Gattuso, M.; Perez, P.; Zacchino, S. Evidence for the mechanism of action of the antifungal phytolaccoside B isolated from phytolacca tetramera hauman. J Nat Prod 2008,71, 1720-1725.

13. Vergauwen, B.; Pauwels, F.; Vaneechoutte, M.; Van Beeumen, J.J. Exogenous glutathione completes the defense against oxidative stress in haemophilus influenzae. J Bacteriol 2003,185, 1572-1581.

14. Patankar, M.; Bhargava, A.; Ahluwalia, A. Comparison of the minimum fungicidal concentration of clotrimazole, ketoconazole, miconazole and terbinafine against clinical isolates of dermatophytes. Indian Medical Gazette 2014, 4.

15. Wei, G.J.; Liang, L.; Cao, C.W.; Zheng, D.Y.; Lin, B. Analysis of superficial mycosis and pathogensin 10042 cases. Chinese Journal Mycology 2017, 12, 6.

16. Wang, L.; Ma, L.; Leng, W.; Liu, T.; Yu, L.; Yang, J.; Yang, L.; Zhang, W.; Zhang, Q.; Dong, J.; Xue, Y.; Zhu, Y.; Xu, X.; Wan, Z.; Ding, G.; Yu, F.; Tu, K.; Li, Y.; Li, R.; Shen, Y.; Jin, Q. Analysis of the dermatophyte Trichophyton Rubrum expressed sequence tags. BMC Genomics 2006,7, 255.

17. CLSI. Reference method for broth dilution antifungal susceptibility testing of filamentous fungi: approved standard - M38-A. 2002. 22.

18. Forman, H.J.; Zhang, H.; Rinna, A. Glutathione: overview of its protective roles, measurement, and biosynthesis. Mol Aspects Med 2009, 30, 1-12. 
19. Aguirre, J.; Hansberg, W.; Navarro, R. Fungal responses to reactive oxygen species. Med Mycol 2006, 44, S101-S07.

20. Bankhead, P. Analyzing fluorescence microscopy images with image. 2014.

21. Pinto, E.; Afonso, C.; Duarte, S.; Vale-Silva, L.; Costa, E.; Sousa, E.; Pinto, M. Antifungal activity of xanthones: evaluation of their effect on ergosterol biosynthesis by highperformance liquid chromatography. Chem Biol Drug Des 2011,77, 212-222.

22. Lopes, G.; Sousa, C.; Bernardo, J.; Andrade, P. B.; Valentao, P.; Ferreres, F.; Mouga, T. Sterol profiles in 18 macroalgae of the Portuguese coast. J Phycol 2011,47,1210-1218.

23. Ghannoum, M. A.; Hossain, M. A.; Long, L.; Mohamed, S.; Reyes, G.; Mukherjee, P. Evaluation of antifungal efficacy in an optimized animal model of Trichophyton Mentagrophytes-dermatophytosis. J Chemother 2004, 16, 139-144.

24. Ghannoum, M. A.; Long, L.; Pfister,W. R. Determination of the efficacy of terbinafine hydrochloride nail solution in the topical treatment of dermatophytosis in a guinea pig model. Mycoses 2009, 52, 35-43.

25. Garvey, E. P.; Hoekstra, W. J.; Moore, W. R.; Schotzinger, R. J.; Long, L.; Ghannoum, M. A. Vt-1161 dosed once daily or once weekly exhibits potent efficacy in treatment of dermatophytosis in a guinea pig model. Antimicrob Agents Chemother2015,59, 1992-1997.

26. Leck, A. Preparation of lactophenol cotton blue slide mounts. Community Eye Health 1999,12,24.

27. Ponka, D.; Baddar, F. Microscopic potassium hydroxide preparation. Can Fam Physician2014, $60,57$.

28. Ishijima, S. A.; Hayama, K.; Burton, J. P.; Reid, G; Okada, M; Matsushita, Y.; Abe, S. Effect of streptococcus salivarius $\mathrm{k} 12$ on the in vitro growth of candida albicans and its protective effect in an oral candidiasis model. Appl Environ Microbiol 2012, 78,72190-72199.

29. Rovid Spickler, A. Dermatophytosis ringworm. Tinea 2004.

30. Kahn, J.N.; Hsu, M. J.; Racine, F.; Giacobbe, R.; Motyl, M. Caspofungin susceptibility in aspergillus and non-aspergillus molds: inhibition of glucan synthase and reduction of betad-1,3 glucan levels in culture. Antimicrob Agents Chemother2006, 50 , 2214-2216.

31. Steinbach, W.J.; Cramer, R.A.; Perfect, B.Z.; Henn, C.; Nielsen, K.; Heitman, J.; Perfect, J.R. Calcineurin inhibition or mutation enhances cell wall inhibitors against aspergillus fumigatus. Antimicrob Agents Chemother 2007, 51, 2979-2981.

32. Hiroyasu, K. Animal model for superficial mycosis.Nippon Ishinkin Gakkai Zasshi2009, 50, 8589.

33. Yu, L.; Zhang, W.; Wang, L.; Yang, J.; Liu, T.; Peng, J.; Leng, W.; Chen, L.; Li, R.; Jin, Q. Transcriptional profiles of the response to ketoconazole and amphotericin B in Trichophyton Rubrum. Antimicrob Agents Chemother2007, 51, 144-153.

34. Muller, C.; Binder, U.; Bracher, F.; Giera, M. Antifungal drug testing by combining minimal inhibitory concentration testing with target identification by gas chromatography-mass spectrometry. Nat Protoc 2017, 12 , 947-963.

35. Persinoti, G.F.; de Aguiar Peres, N. T.; Jacob, T.R.; Rossi, A.; Vencio, R.Z.; Martinez-Rossi, N.M. RNA-sequencing analysis of Trichophyton Rubrum transcriptome in response to sublethal doses of acriflavine. BMC Genomics $2014,15$.

36. Souza, B.D.; Sartori, D.S.; Andrade, C.; Weisheimer, E.; Kiszewski, A.E. Dermatophytosis caused by Microsporum Gypseum in infants: report of four cases and review of the literature. An Bras Dermatol 2016, 91 ,823-825.

37. Thorpe, G.W.; Fong, C.S.; Alic, N.; Higgins, V.J.; Dawes, I.W. Cells have distinct mechanisms to maintain protection against different reactive oxygen species: oxidative-stress-response genes. Proc Natl Acad Sci USA 2004,101, 6564-6569.

38. Calderon, R.A.; Shennan, G.I. Susceptibility of Trichophyton Quinckeanum and Trichophyton rubrum to products of oxidative metabolism. Immunology 1987, 61,283-288.

39. Martinez, D.A.; Oliver, B.G.; Graser, Y.; Goldberg, J. M.; Li, W.; Martinez-Rossi, N.M.; Monod, M.; Shelest, E.; Barton, R.C.; Birch, E.; Brakhage, A.A.; Chen, Z.; Gurr, S. J.; Heiman, D.; Heitman, J.; Kosti, I.; Rossi, A.; Saif, S.; Samalova, M.; Saunders, C.W.; Shea, T.; Summerbell, R.C.; Xu, J.; Young, S.; Zeng, Q.; Birren, B.W.; Cuomo, C.A.; White, T.C. 
Comparative genome analysis of Trichophyton rubrum and related dermatophytes reveals candidate genes involved in infection. MBio 2012, 3, e00259-12.

40. Namasivayam, S.; Sher, A.; Glickman,M. S.; Wipperman, M. F.The microbiome and tuberculosis: early evidence for cross talk. MBio 2018, 9 .

41. Ferreira, M.E.; Colombo, A.L.; Paulsen, I.; Ren, Q.; Wortman, J.; Huang, J.; Goldman, M.H.; Goldman, G.H. The ergosterol biosynthesis pathway, transporter genes, and azole resistance in Aspergillus Fumigatus. Med Mycol 2005, 43,313-319.

42. Pocsi, I.; Prade, R.A.; Penninckx, M.J. Glutathione, altruistic metabolite in fungi. Adv Microb Physiol 2004,49, 1-76.

43. Vergauwen, B.; Pauwels, F.; Van Beeumen, J.J.Glutathione and catalase provide overlapping defenses for protection against respiration-generated hydrogen peroxide in haemophilus influenzae. J Bacteriol 2003, 185,5555-5562.

44. Marinho, H.S.; Real, C.; Cyrne, L.; Soares, H.; Antunes, F. Hydrogen peroxide sensing, signaling and regulation of transcription factors. Redox Biol2014, 2, 535-562.

45. Schmit, J.C.; Brody, S. Biochemical genetics of neurospora crassa conidial germination. Bacteriol Rev 1976,40, 1-41.

46. Bienert, G.P.; Schjoerring, J.K.; Jahn, T.P. Membrane transport of hydrogen peroxide. Biochim Biophys Acta 2006,1758, 994-1003.

47. Achterman, R.R.; Smith, A.R.; Oliver, B.G.; White, T.C. Sequenced dermatophyte strains: growth rate, conidiation, drug susceptibilities, and virulence in an invertebrate model. Fungal Genet Biol 2011, 48,335-341.

48. Simons, V.; Morrissey, J.P.; Latijnhouwers, M.; Csukai, M.; Cleaver, A.; Yarrow, C.; Osbourn, A. Dual effects of plant steroidal alkaloids on Saccharomyces Cerevisiae. Antimicrob Agents Chemother 2006, 50,2732-2740.

49. Tian, J.; Yu, J.; Ge. Y.; Qi, L.; Li, C. Effect of Macleaya Cordata alkaloids on five skin fungus. Chinese Traditional Patent Medicine 2010, $7,4$.

50. Onishi, J.; Meinz, M.; Thompson, J.; Curotto, J.; Dreikorn, S.; Rosenbach, M.; Douglas, C.; Abruzzo, G.; Flattery, A.; Kong, L.; Cabello, A.; Vicente, F.; Pelaez, F.; Diez, M.T.; Martin, I.; Bills, G.; Giacobbe, R.; Dombrowski, A.; Schwartz, R.; Morris, S.; Harris, G.; Tsipouras, A.; Wilson, K.; Kurtz, M.B. Discovery of novel antifungal (1,3)-beta-d-glucan synthase inhibitors. Antimicrob Agents Chemother 2000, 44 , 368-377.

51. Ji, H.; Zhang, W.; Zhang, M.; Kudo, M.; Aoyama, Y.; Yoshida, Y.; Sheng, C.; Song, Y.; Yang, S.; Zhou, Y.; Lü, J. Journal of Medicinal Chemistry 2003,46, 474-485.

52. Ngo, M. A.; Maibach, H. I.Dermatotoxicology: historical perspective and advances. Toxicol Appl Pharmacol 2010, 243,225-238.

53. Shimamura, T.; Kubota, N.; Shibuya, K. Animal model of dermatophytosis. J Biomed Biotechnol 2012, 2012,125384.

54. Song, X.; Wei, Y. X.; Lai, K. M.; He, Z. D.; Zhang, H. J. In vivo antifungal activity of dipyrithione against Trichophyton Rubrum on guinea pig dermatophytosis models. Biomed Pharmacother 2018,108, 558-564.

55. Agarwal, A. K.; Rogers, P. D.; Baerson, S. R.; Jacob, M. R.; Barker, K. S.; Cleary, J. D.; Walker, L. A.; Nagle, D. G.; Clark, A. M. Genome-wide expression profiling of the response to polyene, pyrimidine, azole, and echinocandin antifungal agents in Saccharomyces Cerevisiae.J Biol Chem 2003, 278, 34998-35015. 